\title{
The impact of dietary intake and exercise on metabolic health in Saudi Arabian women
}

\author{
A. Alhajri, F. McCullough and A.M. Salter \\ Division of Nutritional Sciences, School of Biosciences, University of Nottingham, Sutton Bonington, LE125RD
}

Like much of the Middle East, Saudi Arabia is seeing a dramatic increase in obesity, particularly amongst women ${ }^{(1)}$. Obesity is frequently associated with the development of metabolic syndrome (MS) and associated risk of developing type 2 diabetes and cardiovascular disease. For females, MS is defined by the International Diabetes Federation (IDF) as including the following factors: Waist Circumference (WC) $\geqslant 80 \mathrm{~cm}$, Systolic Blood Pressure $\geqslant 130 \mathrm{mmHg}$, fasting plasma triacylglycerol (TAG) $\geqslant 1.7 \mathrm{mmol} / \mathrm{l}$, High Density Lipoprotein (HDL) cholesterol $<1.29 \mathrm{mmol} / 1$, fasting plasma glucose $\geqslant 5.6 \mathrm{mmol} / \mathrm{l}^{(2)}$. Sedentary behaviours, persistent low levels of physical exercise and poor cardiorespiratory fitness (CRF) may be further risk factors for MS ${ }^{(3)}$. In Saudi Arabian females cultural factors may specifically limit the amount of physical activity undertaken. This study examined the effect of dietary intervention, without (D) or with $(\mathrm{D}+\mathrm{E})$ changes in physical activity, in 40 obese (BMI $\left.(\mathrm{Mean} \pm \mathrm{SD})=36 \cdot 5 \pm 5 \cdot 1 \mathrm{~kg} / \mathrm{m}^{2}\right) \mathrm{Saudi}$ Arabian women aged 18-60y.

The study was approved by the ethics committee of King Faisal University, Saudi Arabia. All subjects were provided with dietary advice, including follow-up using regular 'WhatsApp' messages, focusing on dietary goals for reducing energy intake and adopting a 'healthy diet'. The D + E group also attended a fitness centre six times per week where they undertook approximately 20 min vigorous and 40 min moderate exercise.

Total dietary energy intake was reduced by $41 \%$ and $35 \%$ for D and D + E groups respectively. MS associated risk factors were measured prior to and after intervention and are shown in the table. Data was analysed by repeated measures ANOVA.

\begin{tabular}{|c|c|c|c|c|c|c|}
\hline & \multicolumn{2}{|l|}{ Diet Only } & \multicolumn{2}{|c|}{ Diet + Exercise } & \multicolumn{2}{|c|}{ ANOVA (p-value) } \\
\hline & Before & After & Before & After & Time & Time $\times$ intervention \\
\hline $\mathrm{WC}(\mathrm{cm})$ & $98.9 \pm 1.9$ & $92.6 \pm 11.9$ & $99.9 \pm 12 \cdot 7$ & $95 \cdot 2 \pm 2 \cdot 0$ & $<0.001$ & 0.235 \\
\hline $\mathrm{SBP}(\mathrm{mmHg})$ & $117 \pm 13$ & $112 \pm 13$ & $119 \pm 10$ & $114 \pm 10$ & 0.008 & 0.894 \\
\hline Glucose & $4.9 \pm 0.7$ & $4.6 \pm 0.7$ & $4.8 \pm 0.7$ & $4.9 \pm 0.7$ & 0.622 & $0 \cdot 169$ \\
\hline TAG & $0.9 \pm 0 \cdot 3$ & $0.7 \pm 0.3$ & $0.9 \pm 0.5$ & $0 \cdot 8 \pm 0.4$ & $0 \cdot 017$ & $0 \cdot 541$ \\
\hline HDL chol & $1.27 \pm 0.29$ & $1 \cdot 24 \pm 0 \cdot 30$ & $1 \cdot 24 \pm 0.32$ & $1.47 \pm 0.39$ & $0 \cdot 119$ & $0 \cdot 040$ \\
\hline
\end{tabular}

Glucose, TAG \& HDL chol all in $\mathrm{mmol} / 1$

Both groups of women clearly displayed WC levels that would place them at risk of developing MS. However, of the other risk factors, only HDL chol fell into the range specified within the IDF MS definition. Following dietary intervention WC, SBP and plasma TAG were all significantly reduced independently of exercise inclusion. HDL chol showed a specific response to exercise, increasing in the $\mathrm{D}+\mathrm{E}$ group but not the $\mathrm{D}$ group.

This study shows that, despite having a WC within the range that increases risk of MS, these Saudi Arabian women displayed few other symptoms of MS. However, reduced energy intake, and weight loss, considerably improved their metabolic profile independently of whether this was accompanied by increased physical activity.

A.A. was supported by a PhD studentship from the Saudi Arabian Government

1. Al-Daghri N., Alkharfy K., Al-Attas O., et al. (2014) BMC Cardiovasc Disorders, 14:51.

2. IDF Taskforce on Epidemiology and Prevention (2006) The IDF consensus worldwide definition of the Metabolic Syndrome, IDF Communications. 3. Steele RM, Brage S, Corder K, Wareham NJ, Ekelund U. (2008) J Appl Physiol. 105:342-351. 